\title{
Optimization of Highly-Structured Mechanical Systems with Abnormal Functioning through the Example of a Precipitation Centrifugation of Powder Precipitates
}

\author{
M.A. Kuzin", O.I. Dreganov \\ JSC "State Scientific Center - Research Institute of Atomic Reactors", 433510 Dimitrovgrad-10 \\ *Corresponding Author: disputfuzzy@gmail.com
}

Copyright (C) 2013 Horizon Research Publishing All rights reserved.

\begin{abstract}
A model of vibration reliability for a vertical submersible centrifuge was suggested that is used in optimization of centrifugation. A set of software tools was developed related to simulation modeling and centrifuge computation. The plotted model and software can be applied for optimization of centrifuging devices and processes, nuclear fuel cycle systems and processes.
\end{abstract}

Keywords Submersible Centrifuge, Model, Critical Frequency, Centrifuging, Vibration Reliability

\section{Introduction}

The mathematical and simulation modeling [1-3] play an important role in the study of the performance, reliability and service life of centrifuges used for the precipitation processes.

A submersible centrifuge is a combination of subsystems depended both on each other and random external factors. Sometimes, it is impossible to carry out an analytical research of processes occurring when operating such devices as centrifuges. The most effective way out here is to use stochastic simulation models. In this connection, a mathematical model presented in this paper is urgent and of applied importance.

The object of examination is a vertical submersible centrifuge used to separate suspensions. Models and methods to assess the performance, reliability, service life and efficiency of the vertical submersible centrifuge are the subject of examination.

The purpose of work is to define an optimal rotation frequency of the vertical submersing centrifuge shaft. To reach the goal, a process of precipitation centrifuging is considered from the viewpoint of the vibration reliability; mathematical models of the vibration reliability and precipitation centrifuging have been plotted. To implement the above-said models, the well-known Langevin equation numerical method was modified by means of a minimax criterion. The vibration reliability model was plotted at the first stage of study [4]. This paper presents the results of the plotting of the precipitation centrifuging method, development of the modified numerical method, implementation of the computational algorithm and verification of the plotted models.

\section{Methodology}

The Dunkerley method was used to plot the models and calculate the vibration reliability parameters [1-2, 4]; the Ornstein - Uhlenbeck process [5] as well as the minimax criterion were used to determine the optimal rotation frequency of the vertical submersing centrifuge shaft. The adequacy of the model was checked by comparing the data of the in-situ test and computational experiment as well as empirical distribution function based on the generated data.

In this study, the mass of collected precipitate $\Phi_{r}$ in the in-situ test was determined using the gravimetrical method by the mass of tungsten carbide particles precipitate collected in distilled water and dried.

\section{Results}

The centrifuge shaft has two bearings, a collector being fixed on the free shaft end over the bar. To simplify the calculations, the bar and the collector are presented as a cylinder with diameter equal to the outer diameter of the collector. The calculation model of the system rotating centrifuge shaft - bar - collector is shown in Fig. 1 [1-3]. Based on the results of the examination of centrifugal vibration reliability $[1-3]$, a mathematical model of precipitation centrifuging is plotted to determine the mass of collected precipitate $\Phi$ taking into account a practically observed decrease in the centrifuge performance due to the random deviation of the mass center from the rotation axis. 
The optimal rotation frequency is determined from the condition for the minimum of the maximal values $\Phi_{t}$.
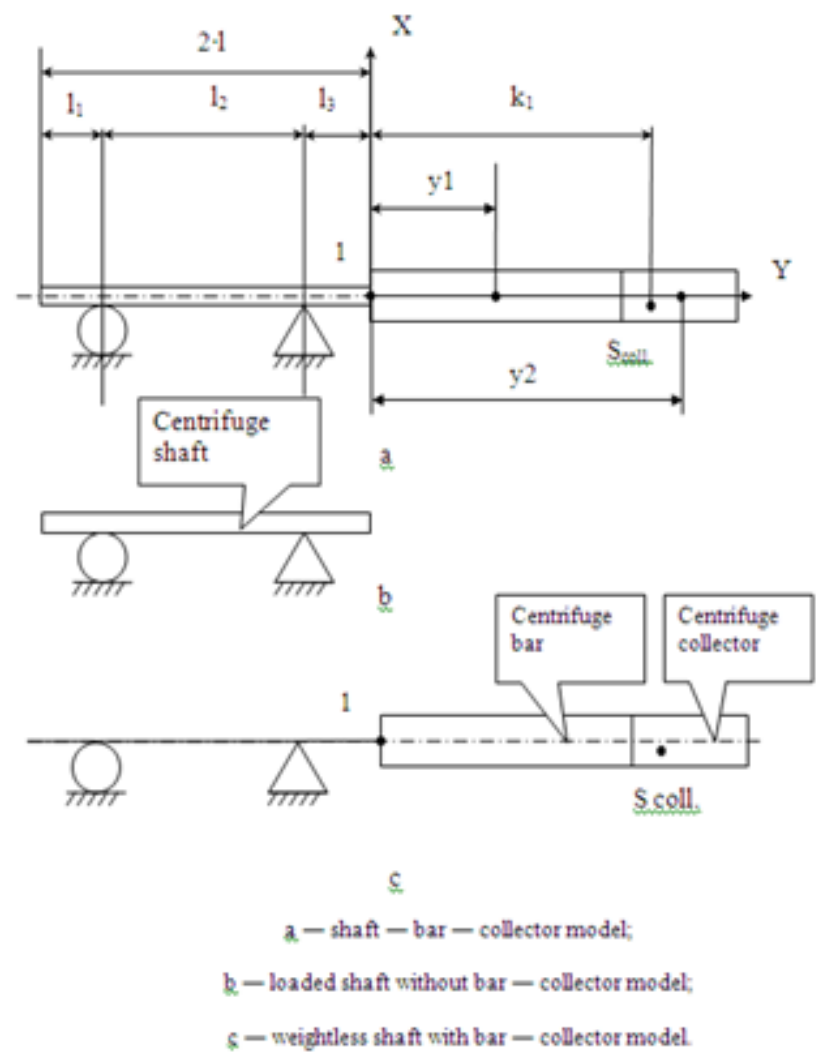

Figure 1. Shaft - bar - collector calculation model

Function $\Phi_{t}$ is defined as a product of perfect centrifuge performance (without deviation from the mass center) $Y$ and collection fullness coefficient $X=\left(X_{t}\right)_{0 \leq t \leq T}$. The Ornstein Uhlenbeck process can be introduced as $X_{t}$ (with $X_{0}-\mathcal{F}_{0}$ -measured Gaussian random variable with distribution $\mathcal{N}(0 ; 1))$. Then, the considered mathematical model of precipitation centrifuging can be described through the system of equations [6]:

$$
\left\{\begin{array}{l}
\Phi_{t}=\varepsilon_{0} \cdot Y \cdot \overline{X_{t}} \\
Y=\varepsilon_{1} \cdot R_{2}{ }^{3} \cdot \omega^{1+\delta}, \delta \geq 0, R_{2}>0 \\
\overline{X_{t}}=X_{\min \{t ; \tau\}} \\
X_{t}=e^{-\lambda_{x} \cdot s_{\omega} \cdot t}\left(X_{0}+\int_{0}^{t} e^{\lambda_{x} \cdot s_{\omega}} \sigma_{x} d W_{t}\right), \lambda_{x}>0, s_{\omega} \geq 0, \sigma_{x} \geq 0 \\
\tau=\inf \left(t: t>0, X_{t}=0\right)
\end{array}\right.
$$

where $\Phi_{t}-$ mass of collected precipitate, $\mathrm{kg}$;

$Y$ - centrifuge performance, $\mathrm{kg} / \mathrm{s}$;

$X=\left(X_{t}\right)_{0 \leq t \leq T}-$ collection fullness coefficient, $X_{0}=0,5$;

$\tau=\inf \left(t: t>0, X_{t}=0\right)$ - time moment $t$, at which value $X_{t}$ achieves $X_{t}=0$ for the first time;

$s_{\omega}$ - displacement coefficient $S, \mathrm{~m} / \mathrm{s}^{2}: s_{\omega}=\omega^{2} \cdot S$;

$t \in[0 ; T]-$ precipitation centrifuging duration, s;

$\varepsilon_{0,1}, \delta, \lambda_{x}, \sigma_{x}-$ empiric constants,

$W=\left(W_{t}\right)_{0 \leq t \leq T}-$ standard Wiener process.

As the Ornstein - Uhlenbeck process is the solution to the Langevin equation [5] in the given conditions, the system (1) with regard to the implementation of the random computation processes can be presented as

$$
\left\{\begin{array}{l}
\Phi_{t}=\varepsilon_{0} \cdot Y \cdot \overline{X_{t}} \\
Y=\varepsilon_{1} \cdot R_{2}^{3} \cdot \omega^{1+\delta}, \delta \geq 0, R_{2}>0 \\
\overline{X_{t_{k}}}=X_{\min \left\{t_{k} ; \tau\right\}} \\
d X_{t}=-\lambda_{x} \cdot s_{\omega} \cdot X_{t} d t+ \\
\sigma_{x} d W_{t}, \lambda_{x}>0, s_{\omega} \geq 0, \sigma_{x} \geq 0 \\
\tau=\inf \left(\boldsymbol{t} . \boldsymbol{t}>0, \boldsymbol{X}_{t}=0\right),
\end{array}\right.
$$

A shift from (1), (2) to a discrete model is divided into two stages. The first stage comprises the replacement of continuous area $0 \leq t \leq T$ with a discrete one - total finite number of points $n \in \mathrm{N}, T \in \mathrm{R}^{+} \quad$ : $\left\{t_{k}: t_{k}=t_{k-1}+\Delta_{k}, k=\overline{1, n}\right\}$, where $t_{0}=0, t_{n}=T ; \Delta_{k}-$ sampling rate that meets condition $\left|X_{t_{k-1}}-X_{t_{k}}\right|<0,05$. The second stage of the shift from a continuous model includes generating discrete analogues of differential equations (replacement of the derivatives with the corresponding finite differences). Thus, for each $\omega, k$ and $\xi_{i}, i=0,1 \ldots-$ independent Gaussian random variables on $(\Omega, F, P)$ such that $E \xi_{i}=0, E \xi_{i}^{2}=1$ the following approximation can be possible:

$$
\left\{\begin{array}{l}
\Phi_{t_{k}}=\varepsilon_{0} \cdot Y \cdot \overline{X_{t_{k}}} \\
Y=\varepsilon_{1} \cdot R_{2}^{3} \cdot \omega^{1+\delta}, \delta \geq 0, R_{2}>0 \\
\overline{X_{t_{k}}}=X_{\min \left\{t_{k} ; \tau\right\}} \\
\eta_{k}=\sum_{i=1}^{12} \xi_{i}-6 \\
X_{t_{k+1}}=X_{t_{k}}\left(1-\lambda_{x} \cdot s_{\omega} \cdot \Delta_{k}\right)+\sigma_{x} \cdot \eta_{k} \cdot \sqrt{\Delta_{k}} \\
\tau=\inf \left(t_{k}: t_{k}>0, X_{t_{k}}=0\right) .
\end{array}\right.
$$

The optimal rotation frequency corresponds to the minimal one of the maximum values of collection mass $\Phi$ in all the range of rotation frequencies up to the first critical frequency determined by the Dunkerley method [6].

The computational algorithm giving the numerical solution to the task $(1-3)$ for the vertical submersible centrifuging system is done in software "Reliability 2002" [6, 7]. For the target parameters of the centrifuging system 
programs compute the first critical rotation frequency by the Dunkerley method and the mass of collected precipitate using software "Computation of the optimal frequency".

The adequacy of the plotted model was checked by comparing the data of computational experiment (Fig. 2) done using software "Reliability 2002" and in-situ test (Fig. 3 ) of the vertical submersible centrifuge as well as empirical distribution function based on the generated data (Fig. 4).

\section{Discussion}

As seen from the data given in Fig. 2-4, the results of the computations agree well with the in-situ test data. Check of the model adequacy by empirical distribution functions $\Phi$ (Fig. 4) for three collection rate values showed that the computation data agreed well with the in-situ test. The maximal discrepancy value determined by the sum of squared difference of the values of empirical distribution functions $\Phi$ of in-situ test and computational experiment made up 0.03 ( 0.03 for $\omega=105 \mathrm{rad} / \mathrm{s}$ and $207 \mathrm{rad} / \mathrm{s}, 0.01$ for $\omega=157 \mathrm{rad} / \mathrm{s})$.

The optimal rotation frequency $\omega_{\text {opt }}$ determined from the condition of the minimum of maximal values of collection mass $\Phi$ according to the in-situ test experimental data and computations made up $157 \mathrm{rad} / \mathrm{s}$. Thus, a value of $157 \mathrm{rad} / \mathrm{s}$ can be recommended as the upper limit of the operating rotation frequency range, which is 0.7 of the first critical frequency value determined by the Dunkerley method.

The study revealed that the range of rotation frequencies, the upper limit of which makes up no more than 0.7 of the first critical frequency value determined using the Dunkerley method, can be recommended for the vertical submersible centrifuge considered in this paper.

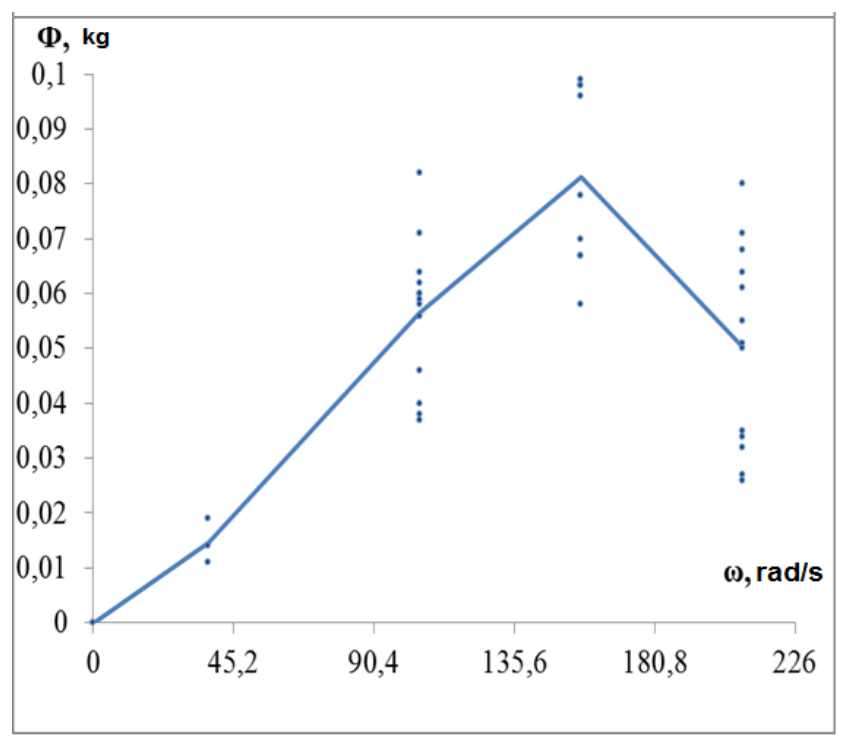

Figure 2. In-situ test results

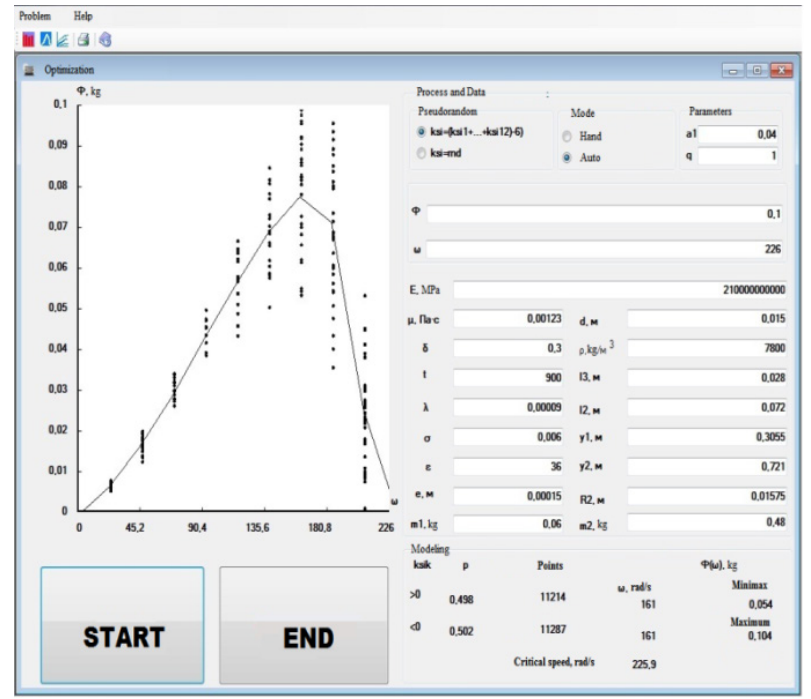

"Reliability 2002"

Figure 3. Computation experiment results in software
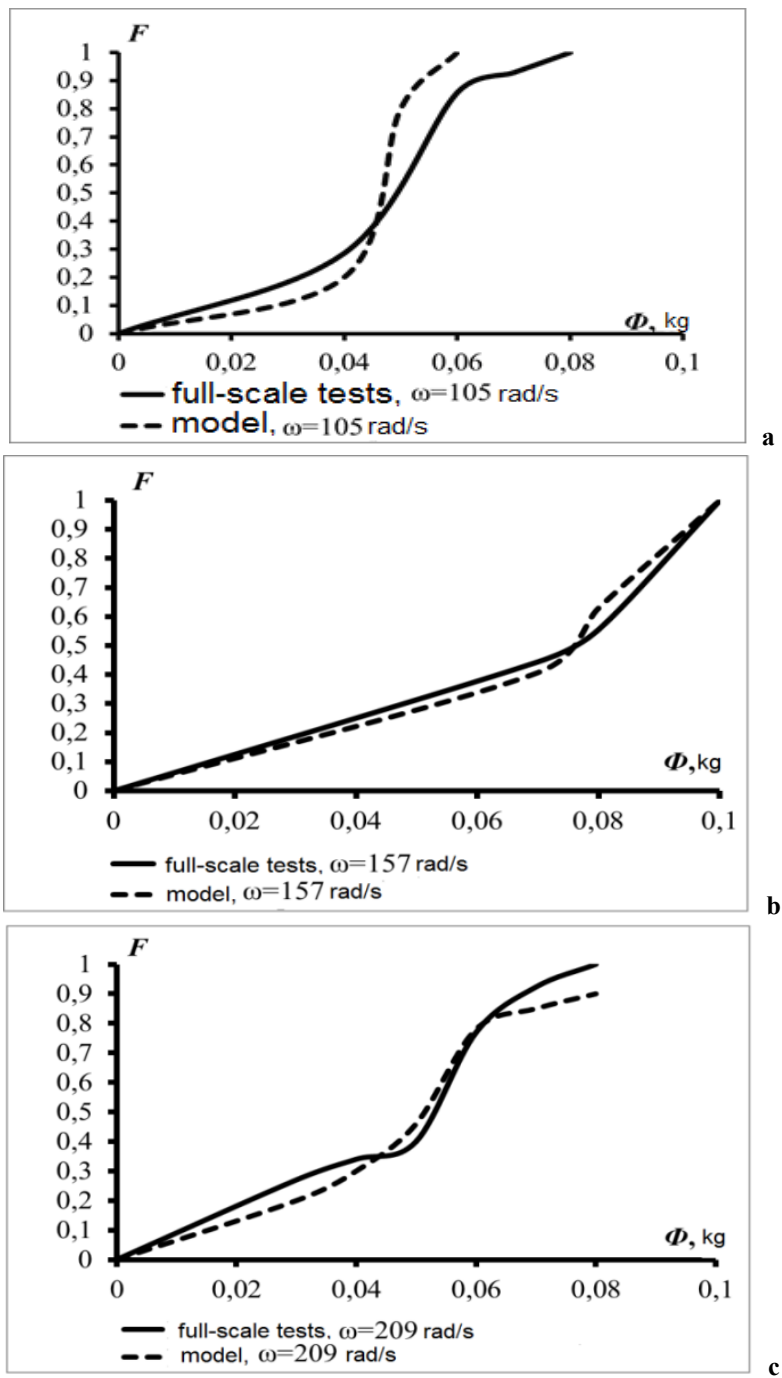

$\mathrm{a}-\omega=105 \mathrm{rad} / \mathrm{s}, \mathrm{b}-\omega=157 \mathrm{rad} / \mathrm{s}, \mathrm{c}-\omega=209 \mathrm{rad} / \mathrm{s}$

Figure 4. Empirical distribution functions according to the in-situ test and computation experiment data 


\section{Conclusion}

The results of examination of various centrifuge characteristics, phenomena and processes presented in this paper are of practical interest in designing, operation of new and upgrading of the known centrifugal devices and mechanisms that are used in chemical reprocessing, nuclear fuel cycle processes and systems. The obtained data allows a more accurate understanding of the nature of occurring processes related to suspension separation and removal of precipitates from chemical reactors.

\section{REFERENCES}

[1] Kuzin, M.A. Simulation and Vibrational Reliability of Centrifuge for Separating Suspensions // Russian Engineering Research ISSN 1068-798X. Allerton Press, Inc.: Vol. 29, No. 11, 2009. p.1099-1101. DOI:10.3103/S1068798X09110069.

[2] Kuzin, M.A. Vibration reliability and endurance of a centrifuge for separating suspensions // Chemical and Petroleum Engineering. ISSN 0009-2355 (Print) 1573-8329
(Online). - Springer Science+Business Media, Inc: Vol. 46, Nos. $1-2,2010 . \quad$ pp. 45-50. DOI: $10.1007 / \mathrm{s} 10556-010-9287-8$.

[3] Kuzin, M.A., Dreganov, O.I. Optimization of Highly-Structured Mechanical Systems with Abnormal Functioning through the Example of the Precipitation Centrifugation of Powder Precipitates in Molten Salt. VII Russian Conference on Radiochemistry "Radiochemistry 2012". - P.146.

[4] Kuzin, M.A. Model vibratsionnoy nadezhnosti tsentrifugi [The Model of Vibration Reliability of a Centrifuge]. Vestnik mashinostroyeniya, no.8(2012):11-12. http://www.mashin.ru/eshop/journals/vestnik_mashinostroen iya/2012/08/

[5] Butov, A.A., Ravodin, K.O. Theory of Random Processes: Teaching Manuals. - Ulyanovsk: U1SU, 2009. - 62p.

[6] Kuzin, M.A. Simulation of Destruction Processes of the Partly Observed Centrifuging System: Thesis ... Ph.D. in Engineering - Ulyanovsk, 2012. - 133 p.

[7] Reliability $2002 /$ (Ulyanovsk Technical State University). - No.2002611045; Application request No.2002610807 as of 6.05.2002 // Computer Software Registry. - 2002. M.A. Kuzin, S.M. elinis. 